\title{
Challenges in Deploying a Delay Tolerant Network
}

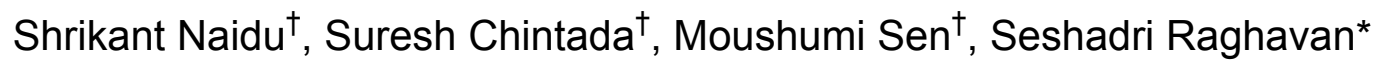 \\ ${ }^{\dagger}$ Motorola Research Labs, Motorola India \\ \{shrikant, suresh.chintada, moushumi\}@motorola.com \\ *Mobile Devices, Motorola India \\ seshadri.r@motorola.com
}

\begin{abstract}
Delay Tolerant Networking (DTN) is the area of networking that addresses challenges in disconnected, disrupted, and networks without end-to-end connection. DTN has been an active area of research in the recent past; there have been attempts to use concepts from DTN to extend the reach of networks to remote rural areas as well. MotoPost ${ }^{\mathrm{TM}}$ is a solution that is designed to establish a communications framework for rural communities in remote areas using non-real-time store and forward mechanisms and draws inspiration from ongoing research in this area. The concept was developed to address the communication and information access needs of remote rural villages in India which do not have access to communication technologies, but can be extended to other similar locations globally. MotoPost $\mathrm{t}^{\mathrm{TM}}$ is based on DTN where dual mode mobile phones are used as data mules to establish an end-to-end data network, we believe this is one of the first attempts to use mobile phones as data mules. In our system we use SMTP as the base application protocol to transfer multimedia messages between MotoPost ${ }^{\mathrm{TM}}$ nodes. In this paper, we present and discuss findings from a limited field deployment of MotoPost ${ }^{\mathrm{TM}}$ in rural India. We will specifically focus on the issues related to deploying specific applications and their demands on such a network, including network provisioning and setup as well as economical and technical feasibility for a large scale deployment. The results of the field trial also form the basis for our recommendations on future directions and research issues in this exciting area of research.
\end{abstract}

\section{Categories \& Subject Descriptors}

C.2.1 [Computer-Communication Networks] Network

Architecture and Design - Network Communications, Store and forward networks, Wireless Communication.

\section{General Terms}

Human factors, Measurement, Design, Reliability, Experimentation.

\section{Keywords}

Delay tolerant network, asynchronous, wireless, rural communication, mobile, addressing, provisioning, routing

\section{INTRODUCTION}

People have been dealing with disconnections in network for applications like email, RFID, and meter reading-instances where the device or end user is not always connected-and have come up with custom applications. DTN Research Group attempted to formalize the use of such networks by building

Permission to make digital or hard copies of all or part of this work for personal or classroom use is granted without fee provided that copies are not made or distributed for profit or commercial advantage and that copies bear this notice and the full citation on the first page. To copy otherwise, or republish, to post on servers or to redistribute to lists, requires prior specific permission and/or a fee.

CHANTS'08, September 15, 2008, San Francisco, California, USA.

Copyright 2008 ACM 978-1-60558-186-6/08/09...\$5.00. overlays which more than supplants the TCP/IP protocols [4]. In general DTN provides a solution to enable networking to nodes that may not have an end to end data communication link [3].

There have been instances of using this type of a network to extend communication or provide connectivity to remote rural areas, with DakNet [6] and KioskNet [8] being two such well known efforts. The DakNet helped break some of the myths related to providing communication to connectivity challenged and economically backward rural areas of India. The DakNet project helped establish some of the earliest insights into providing alternative affordable communication infrastructure based on a DTN paradigm. A key insight from this project states that the benefit of providing everyone access to digital messaging (voice mails, email etc.,) outstrips the advantage of having one or two common public telephones in a typical Indian rural village setting. The project also dispels the myth of poor people having no use of computers and goes on to suggest that given the right price point, the poor are willing to consume digital services to counter poor infrastructure, unfair pricing and corruption. The DakNet experiment also proved that asynchronous communication is sufficient to meet most communication needs of rural communities. Our own field trial validated most of the above observations and provided us additional insights into factors that influence deployment of such challenged networks.

In KioskNet [8], Seth et. al. built upon DakNet [6], extended the DTN architecture and addressed issues that included naming, addressing, forwarding, routing, identity management, application support and security. Some of the design principles included low cost systems, reliability in the face of power outages, disconnection tolerance, user mobility, data privacy, interoperability with internet applications, use of any type of network transport and support for both shared Kiosk and personal PDA \& laptop users. The guiding principles that led to the architecture include 1) shared infrastructure lead to lower costs, 2) self describing data on store-and-forward nodes, 3) decouple address and location - users are mobile, 4) opportunistic networking - use the network that is available, 5) keep control \& data planes separate, 6) use disconnection aware applications or proxies on the Kiosk, 7) increase reliability via data replication.

MotoPost ${ }^{\mathrm{TM}}$ has motivations similar to Daknet and KioskNet and, we believe, is the first system to use mobile phones as data mules. The effort specifically looked into defining application protocols, provisioning mechanisms and simple routing schemes to enable use of mobile devices as data mules, in addition it also explored user feedback and design concepts which are very essential in understanding the deployment, uptake and use of such a solution. The system was validated in a limited field trial across 5 villages in rural India during the spring 2007. MotoPost ${ }^{\mathrm{TM}}$ system architecture takes into account specific needs of people in this region as well as the existing infrastructure to support such a DTN [9]. The livelihood of people in these regions is primarily based on agriculture labor. The literacy levels enable them to converse (speech but rarely written) in local languages, have decent sense 
of basic arithmetic necessary for trade and are aware of other forms of communication via TV. While computer literacy amongst this population is low, they are very familiar with certain other consumer electronic gadgets like radio, TV, VCD players etc.

This paper summarizes some of the insights we gained from the field trial with specific discussion on challenges to be addressed for deploying and sustaining a large scale DTN network. The rest of the paper is organized as follows: Section 2 provides a brief overview of the MotoPost ${ }^{\mathrm{TM}}$ system, Section 3 describes the feedback from the field trial, Section 4 describes some of the challenges related to deploying such a system and identifies future research directions in this area, and finally, Section 5 presents our conclusions.

\section{MOTOPOST ${ }^{\text {TM SYSTEM }}$}

MotoPost ${ }^{\mathrm{TM}}$ builds further on the solutions described in [6] and [8] by concentrating on a few key areas and keeping in focus the requirements $\&$ peculiarities of the Indian rural community \& its available infrastructure [1] [2].

Ethnographic studies [7] conducted by Seshagiri et. al. in rural India gave us an understanding of the information needs of people as well as the variance in these requirements as we move between totally unconnected regions and regions with varying degrees of connectivity (e.g., patches of cellular connectivity, a public telephone, etc.,). Based on these studies, certain applications were identified and a voice \& picture enabled messaging application was developed to address some immediate needs for this population on the MotoPost ${ }^{\mathrm{TM}}$ network. The field trial gave us an excellent opportunity to study the acceptance of such an asynchronous communication system in the presence of alternate but relatively expensive communication mechanisms and to also understand the delay tolerance that people are willing to accept.

The existing infrastructure could be roughly categorized into geographical organization, physical road networks and cellular networks. India is geographically divided into several postal zones and a postal code (PIN) becomes a key component of users identity. India has the largest road network in the world crisscrossing more than 600,000 villages and numerous towns and cities. All the national and state highways to a large extent have cellular coverage.

MotoPost ${ }^{\mathrm{TM}}$ architecture leverages these local characteristics defining India in terms of typical network coverage. A dual mode mobile phone is used as a data mule to store and forward information in this network. The dual mode mobile phone also enabled connectivity via two different types of wireless networks, namely $\mathrm{WiFi}^{\mathrm{TM}}$ and cellular (data). The mobile phone is advantageous as it could be hand carried, is battery powered and can potentially be used as a mobile Kiosk (shared device), or a data gathering device amongst other possible advantages.

\subsection{Architecture}

The primary network elements of the MotoPost ${ }^{\mathrm{TM}}$ system include 1) Kiosks with $\mathrm{WiFi}^{\mathrm{TM}}$ access capability located in remote villages, 2) dual mode mobile terminals which are termed as Boxon-Bus (BoB) and have both cellular (data) and $\mathrm{WiFi}^{\mathrm{TM}}$ connectivity and that ride on a physical transport network like buses, motorcycles, bicycles, etc., 3) a Dispatch Center (DC) that serves as the main internet gateway to the MotoPost ${ }^{\mathrm{TM}}$ network, and optionally, 4) Intermediate Caching Points (ICP) which are $\mathrm{WiFi}^{\mathrm{TM}}$ capable and which may also be connected to the internet via the DC using dialup and/or cellular network. Keeping initial and operational costs low was one of the prime considerations for the choice of architecture and components. More details of the system are available in [9].

The MotoPost ${ }^{\mathrm{TM}}$ system implements a unique addressing scheme which allow users to be provisioned into the system at the village operator assisted Kiosks. The Kiosk is a shared device which has $\mathrm{WiFi}^{\mathrm{TM}}$ capability and is used primarily for private and secure access to one's communication services including, composing, sending, receiving and consuming multimedia messages. The Kiosk is run by a local entrepreneur who would help subscribers register for services as well as access registered services for a small fee. The user interface design of the Kiosk takes into account localization needs to cater to people with varying levels of literacy and built upon existing paradigms of communication to improve usability. A registered user on a Kiosk needs to provide a login name and a password to access his communication services. Optionally, there is also a provision to support biometric based authentication system. The Kiosk connects over WiFi ${ }^{\mathrm{TM}}$ to BoBs when they are in close proximity to each other, to exchange messages based on a routing algorithm designed for MotoPost $\mathrm{T}^{\mathrm{TM}}$ [9]. At the beginning of each trip the BoB is provisioned over the air with the transport route of the vehicle carrying it, the routing algorithm uses this route information to decide whether the BoB should hold on to a message or send it to the Dispatch Center (or DC) when it comes into cellular (GPRS) coverage. The messages, once delivered to the Kiosk, are available on the Kiosk until the user deletes the messages. The basic Kiosk is equipped with a web camera, and a headset with microphone and speakers. Other accessories like, printers, etc. could be supported as well.

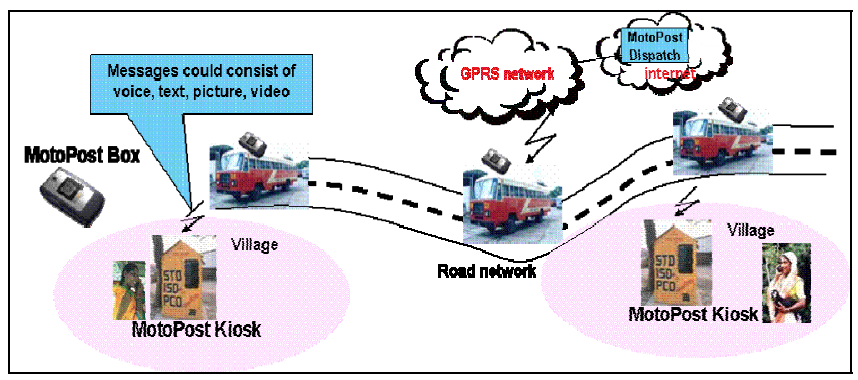

Figure 1. MotoPost ${ }^{\mathrm{TM}}$ Network

The $\mathrm{BoB}$ provides a store and forward information transfer conduit. The BoB could be hand-carried or installed on a physical transport system like a bus or a bicycle, and is used for storing and carrying information in the MotoPost ${ }^{\mathrm{TM}}$ network (Figure 1). BoB communicates with Kiosks and ICPs using WiFi ${ }^{\mathrm{TM}}$, with the DC using cellular (GPRS).

The DC provides the dispatch functionality by facilitating storage and forwarding of information to the destination Kiosk via one or more BoBs or via Internet. It also serves as an internet gateway for MotoPost ${ }^{\mathrm{TM}}$ subscribers, and can route messages, queries and information between the two networks. Apart from this primary function, it is also responsible for provisioning of all Kiosks, ICPs $\&$ BoBs in the system and authentication of the BoBs. The DC also serves as a billing and services portal for Kiosk and $\mathrm{BoB}$ operators. The DC is designed to be either a single physical machine in the cloud, or a network of machines.

An optional element in this network is the ICP - BoBs could use this node as an intermediate data storage point.

\subsection{Addressing}

The trial MotoPost ${ }^{\mathrm{TM}}$ system restricts itself to providing email type delivery of multimedia information. The recipient of a 
message may be another MotoPost ${ }^{\mathrm{TM}}$ subscriber, a cellular user, an Internet email user or a web site (for queries, form submissions, etc.). The "To:" field of the message thus may contain an MS-ISDN number, an email address (either within the known "motopost.in" domain or on the internet), a web page address or a subscriber's postal address.

Seshagiri et. al [7] found most rural residents to be capable of reading \& writing numerals, so we developed a numerical addressing scheme that would be easy to understand and remember. Our addressing scheme relies on the 6 digit Postal Index Number (PIN) which has a built-in (geographic) hierarchy as described in [5]. Each PIN (e.g., "560 093") stands for the smallest unit in the postal services: a delivery post office. Each Kiosk and ICP in the network is identified by a unique 9 digit number that can be decomposed as follows: $<$ Kiosk-ID, PIN $>$ with 3 digits for Kiosk ID within the 6 digit PIN of the postal service area. Each $\mathrm{BoB}$ is uniquely identified by a 14 digit address $<$ BoB-ID, operator-ID $>$, where operator-ID is a unique 6 digit number that identifies a bus operator in the MotoPost ${ }^{\mathrm{TM}}$ system, while the BoB-ID is a 8 digit number unique to each such operator.

Each subscriber is assigned a 14 digit (decimal) MotoPost ${ }^{\mathrm{TM}}$ ID in the following format: <user-ID, Kiosk-ID, PIN>, where the userID is a 5 digit number unique to the Kiosk. The Kiosk application translates this 14 digit MotoPost ${ }^{\mathrm{TM}}$ ID to an email type address as follows: " $<$ user-ID $>@<$ Kiosk-ID $>$. $<$ PIN $>$.motopost.in".

This mechanism of such a hierarchical addressing scheme, based on the geographical location of the Kiosks \& its subscribers, enables use of hierarchical routing algorithms. The other advantage of this choice of addressing is that user administration, and registration do not require any network connectivity. For a description of the routing algorithm used in MotoPost ${ }^{\mathrm{TM}}$ please refer to [9].

\subsection{SMTP}

The application developed for MotoPost ${ }^{\mathrm{TM}}$ trial deployment was internet type mail delivery. Protocols that automatically support mail delivery routing, addressing, user account management were favored. The addressing scheme was modeled after RFC822 which enabled use of POP3 for user account management and addressing. SMTP relay model was chosen as a design alternative to route messages within the network, as the model of mail delivery using SMTP relays in the internet is not very different the requirements of MotoPost ${ }^{\mathrm{TM}}$ system. However, MotoPost ${ }^{\mathrm{TM}}$ system had additional complexity unlike conventional internet systems.

1. The routing tables that SMTP relay nodes used were very dynamic and changed often.

2. All nodes in the system were not of same capability. The BoB's were mobiles with limited processing power and memory. During transfer of messages between BoB \& kiosk or between $\mathrm{BoB} \& \mathrm{DC}$, it was necessary to ensure that message transfer didn't lead to memory full or lack of CPU cycles in the BoB.

Hence, we implemented a control protocol that exchanged routing tables, SMTP message transfer schedule details before routing messages. This combination of a light weight control protocol \& SMTP helped us run the system with no bottlenecks. We also used this protocol to authenticate and provision the MotoPost ${ }^{\mathrm{TM}}$ nodes. Further, ready availability of fully tested SMTP stack in every single device in the MotoPost ${ }^{\mathrm{TM}}$ network reduced the time to develop and deploy a complex network like MotoPost ${ }^{\mathrm{TM}}$. The control protocol was very lightweight and easy to implement.

Extending SMTP to enable other types of internet applications like blogging, web-based queries would be part of the future investigations.

\section{MOTOPOSTTM FIELD TRIAL}

The field trial was conducted during spring 2007 for a period of two weeks wherein 191 local people took part in the trial by registering themselves with the MotoPost ${ }^{\mathrm{TM}}$ system and using it for communicating with their near \& dear in the 5 trial villages. The trial villages met our selection criteria of 1) little or no cellular and/or internet access, 2) socio-economic links to ensure that there would be a need to communicate between these villages, 3) a single local language (Kannada). The identified villages are all located off a State Highway in the southern state of Karnataka, India. These villages were in proximity to each other within an area of $25 \mathrm{sq}$. $\mathrm{Km}$ and about $70 \mathrm{Km}$ from the city of Bangalore. We chose Karnataka state for field trial since the ethnographic studies [7] that led the run up to our project were also conducted in the same state. The User Interface at the Kiosks was designed using the local state language of Kannada. The concept and value proposition of the system was explained to a set of villagers in their local language, and based on their acceptance of the concept and willingness to tryout the system, were recruited to participate in the field trial. These people in turn helped to recruit more people to participate in the field trial. To ensure that there were communication flows across villages, care was taken to ensure that the initial set of people chosen had business or social links with the people in other identified villages in addition we ensured that the set of participants set had some reading/writing skills in the local language, were between the ages 25-50 and had an even representation across men and women.

Table 1. Profile of villages selected for the field trial

\begin{tabular}{|l|l|l|l|l|l|}
\hline Villages & A & B & C & E & D \\
\hline $\begin{array}{l}\text { Public } \\
\text { Phones }\end{array}$ & 6 & 7 & 3 & 15 & 3 \\
\hline $\begin{array}{l}\text { Health } \\
\text { Center }\end{array}$ & No & No & No & Yes & No \\
\hline $\begin{array}{l}\text { Post } \\
\text { Office }\end{array}$ & No & Yes & No & Yes & No \\
\hline $\begin{array}{l}\text { Cellular } \\
\text { coverage }\end{array}$ & No & No & No & Partly & Partly \\
\hline $\begin{array}{l}\text { Dist. to } \\
\text { Highway }\end{array}$ & $18 \mathrm{kms}$ & $\begin{array}{l}23 \mathrm{~km} \\
\mathrm{~s}\end{array}$ & $25 \mathrm{kms}$ & $34 \mathrm{kms}$ & $28 \mathrm{kms}$ \\
\hline Houses & 200 & 300 & 100 & $500+$ & 50 \\
\hline
\end{tabular}

All these villages were small, not particularly well developed in terms of health care, education or communications facilities, and 3 of the 5 villages did not have access to a Post Office also. Further details of these villages, including the number of households in each village and the availability of telephony \& postal services are detailed in Table 1 above. The nearest district headquarters was a Tier III town located $25 \mathrm{Km}$ away from the first village, where both cellular data services (GPRS) \& dialup connection to the internet were available. A point to note is that most of the public phone booths listed in the table above were non-functional, in fact none of the villages had more than 2 public phone booths operating during the period that we conducted the field trial. 
Studies conducted in conjunction with the field trial revealed that about a fifth of the respondents were aware of internet \& email, though very few $(\sim 4 \%)$ had ever used the services. We found that post cards were the primary means of communication followed by telephones (public \& personal phones, both fixed \& cellular) for a majority of respondents $(90 \%)$. When asked to identify the key reasons for communication across different villages \& towns, respondents from the target villages identified social and business needs as the primary factors (see Table 2), whereas only a $3^{\text {rd }}$ of the population expressed satisfaction with the means available today. A primary distrust with the postman (who they believed compromised their privacy while communicating), poor cellular coverage and noise in wireline telephone lines were cited as the key reasons for this dissatisfaction.

Table 2. Key reasons to communicate

\begin{tabular}{|l|l|}
\hline Purpose of communication & $\%$ of respondents \\
\hline Social & 94 \\
\hline Business & 60 \\
\hline $\begin{array}{l}\text { Market rate of farm-related products } \\
\text { for buying \& selling }\end{array}$ & 16 \\
\hline Education & 12 \\
\hline Healthcare \& Family Planning & 9 \\
\hline General News & 5 \\
\hline Weather & 1 \\
\hline
\end{tabular}

\subsection{System Setup}

The backbone of MotoPost ${ }^{\mathrm{TM}}$ messaging system is SMTP, the internet mailer protocol for e-mail messaging. All nodes in the trial MotoPost ${ }^{\mathrm{TM}}$ system: the Kiosks, the BoBs and the DC, run SMTP servers. The messages are routed to the SMTP servers using their domain names. The domain name configuration is based on the Kiosk and BoB addressing schemes described earlier. A light weight control protocol is implemented to wake up SMTP sessions, exchange routing details over SMTP to achieve efficiencies in message transfer. This protocol is also used for provisioning MotoPost ${ }^{\mathrm{TM}}$ nodes. Kiosks run POP3 servers where the user accounts and mailboxes are managed. These are integrated with the corresponding SMTP servers to send and receive email messages. The designed addressing mechanism helped us to leverage SMTP protocol functionality to automatically deliver messages from the $\mathrm{BoB}$ to users registered on the Kiosk. The Kiosk, on the other hand, transfers to the BoB messages only when the Kiosk ID in its destination address matches one of the Kiosks in the current routing table of the BoB.

The Kiosks were laptops with Intel $\mathrm{P} 42.8 \mathrm{GHz}$ processor, $1 \mathrm{~GB}$ RAM and 200GB hard disk space with separate keyboard, optical mouse, webcam, and headset with microphone. Linux ${ }^{\mathrm{TM}}$ OS with a MotoPost ${ }^{\mathrm{TM}}$ Application $(70 \mathrm{~KB})$ and Qmail $(80 \mathrm{~KB})$ was installed on these laptops. The laptops were used during the field trial essentially for ease of transporting as well as for their battery backup. Off-the-shelf indoor WiFi ${ }^{\mathrm{TM}}$ Access Points with a standard transmit power of $100 \mathrm{~mW}$ and external $3 \mathrm{~dB}$ dual antennas were used to provide wireless coverage at each Kiosk.

The DC is a Linux ${ }^{\mathrm{TM}}$ based desktop server running the MotoPost $^{\mathrm{TM}}$ Application Server $(555 \mathrm{~KB})$ along with QMail (2.8MB). The DC is located on a public network and has access to both the internet $\&$ the MotoPost ${ }^{\mathrm{TM}}$ system.

The BoBs used in the trial were a readily available cellular phone with Linux ${ }^{\mathrm{TM}}$ v2.4 OS and in-built $\mathrm{WiFi}^{\mathrm{TM}}$ capability. It has an
ARM 9 RISC processor with $300 \mathrm{MHz}$ clock speed and 64MB memory with a micro SD slot for additional capacity. The 2 major applications were the MotoPost ${ }^{\mathrm{TM}}$ application $(118 \mathrm{~KB})$ and Qmail $(500 \mathrm{~KB})$ on flash. The user space available on flash for message storage was approximately $10 \mathrm{MB}$.

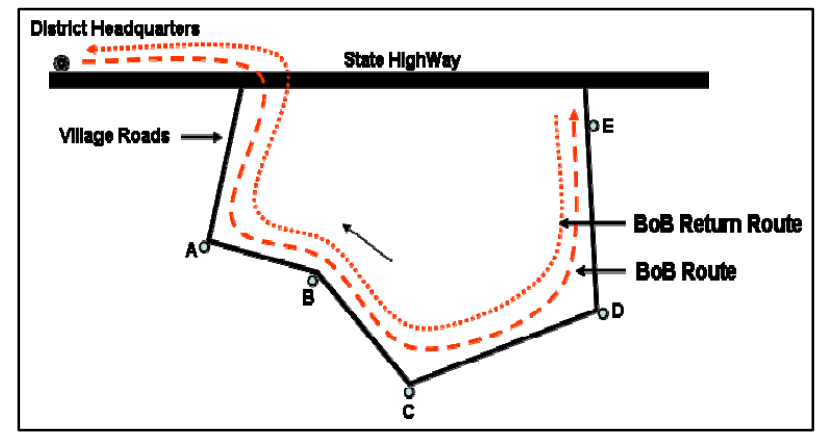

Figure 2. Layout of villages along with BoB Route

A motorcycle was used during the field trial to transport a BoB along the route touching village Kiosks enroute. At the beginning of each day, a BoB was automatically provisioned (over cellular data connection) with its day's route when it established connection with the DC. The BoB also received any undelivered messages lying at DC that it could potentially deliver on its route. In this field trial we used a single route (Figure 2) that traversed the villages in following order (with Kiosk_ID.PINcode): A (001.572101), B (002.572101), C(003.572101), D (001.572140) and finally, E (002.572140) along with a return journey in the reverse order. Finally, at the end of each route (after the BoB had broken connection with the Kiosk at A) all undelivered messages were automatically uploaded onto the DC by the BoB upon connection establishment via cellular data.

The entire network setup for 5 villages, setting up Kiosks, provisioning DC with route, provisioning BoBs took merely half a day including the travel times.

\subsection{User Evaluation and Suggestions}

Both qualitative and quantitative information were collected during the field trial, most of the feedback referenced in this section was obtained during in-depth interviews conducted during the trials.

A total of 191 users of the system, spread over two weeks of trial run, were interviewed with 12 of them who had used the system extensively in-depth. A total of 180 users (94\%) were repeat customers, i.e., they used the MotoPost ${ }^{\mathrm{TM}}$ system 2 or more times during the trial period, and $50 \%$ of these users used it at least once a day for receiving and sending messages.

The field trial validated several conclusions about user experience we had drawn from results of ethnographic studies presented in [7]. The User Interface designed with labels and voiceover instructions in the local language (Kannada) turned out to be extremely successful in its primary goal: ease of usage of the MotoPost $^{\mathrm{TM}}$ system. Almost $80 \%$ of new users to the system found it either "very easy" or "somewhat easy" to learn to use the Kiosk for sending and receiving messages and about $50 \%$ required only minimal help from the Kiosk operators on subsequent use. Additionally, the use of animated icons in a form familiar to the local population (e.g., play/stop/record, letter, post box, green flag/red flag etc.) helped the user to confirm that the actions taken by him/her were indeed the intended ones and 
consequently made them even more confident about their own capabilities and helped bring back users repeatedly to the system.

One observation made that was contrary to a conclusion from ethnographic studies in [7] was the users' ready acceptance of possessing private accounts to store messages, the study had indicated that sharing of resources was common and accepted in the rural community. One conclusion is that such sharing is accepted only when resources are scarce, but is not necessarily by itself desired by the rural community. There was no observation of any sharing of passwords or revealing of personal messages to friends \& neighbors throughout the field trial, after its concept and purpose was explained by the field workers.

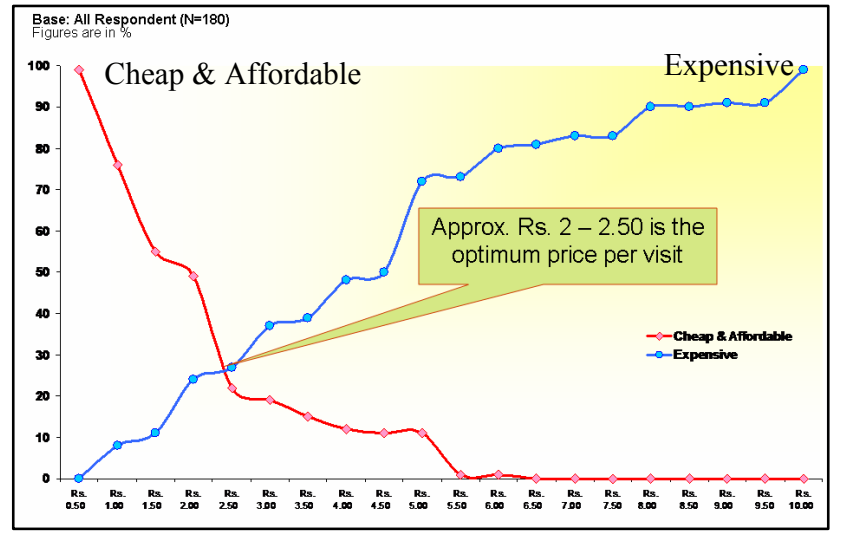

Figure 3. Price Sensitivity Measurement

Lack of prior familiarity with computers did not appear to have caused a hindrance in use of the MotoPost ${ }^{\mathrm{TM}}$ system. On an average, about 15 users per Kiosk composed \& sent approximately 35 messages per day to his/her family and friends in the neighboring villages. Surprisingly, even though the 5 villages under trial system were located within a radius of 25 sq. $\mathrm{Km}$, most of the messages were confined between pairs of villages, leading to a belief that the communities under study were particularly close-knit ones.

Another purpose of the study was to determine if a messaging service such as the one provided by MotoPost ${ }^{\mathrm{TM}}$ would be perceived to be useful to rural communities which have almost negligible communications infrastructure. It was determined from user feedback that such a means is indeed very useful as it saves both time and money to users, with voice $\&$ photo messages being the two most popular features of messaging system used by them. In-depth interviews were conducted to collect information from participants to understand the price points at which such a service would become affordable. Figure 3 depicts the results of the qualitative and quantitative responses that were collected; leading us to conclude that Rs 2.00 to Rs 2.50 (about $5 \not$ to $7 \not$ ) is the optimum price the local population is willing to pay for utilizing the multimedia messaging service.

Several respondents stressed that enhanced communication between their village and the nearby towns where employment \& business opportunities exist would be a very desirable feature. One of the examples cited by the users of the trial system was information about the market rate of vegetables and paddy that would help decide if it was worth making a trip to town for selling their produce. Available employment opportunities each morning by daily wage workers in the neighboring villages $\&$ towns was cited as another example with economic benefit. Access to local daily newspapers and classifieds via messaging were some other examples that the field trial users deemed useful to them.

Messaging was the only application deployed in the field trial. Interviews with the users brought out several other applications categorized in the following application categories:

- Messaging: Between Person to person or Person to Organization. The need here was to be able to send a message to any address within or outside the system. Audio and photos were accepted as the predominant media formats for messaging.

- Information Query System: Obtain near real time information about local jobs, price of commodities and price of produce that they could sell in the market.

- Web Access: Interaction with web servers specifically for job applications, e-governance applications and for submitting forms.

- News Channels: Subscription and consumption of broadcast information related to local and national Business, Politics etc.

Cost and timeliness were major factors that were considered when people mentioned the choice of service that they would use to access these applications. DTN as a technology does not lend itself to real time delivery of services or applications and hence those applications were not considered over such a network. In addition it was also very clear that if people had a synchronous or instantaneous delivery option for a particular application they would prefer that to a typical asynchronous (delay tolerant) approach.

Computer literacy was another barrier in usage of the computer system, even though leveraging known concepts for messaging helped simplify the interface, however other interaction mechanisms for some of the applications mentioned above need to be designed.

The field trial results clearly indicated that there was a need for information access in rural areas both at a local and a global level - the percentage of target population that used the MotoPost ${ }^{\mathrm{TM}}$ system repeatedly to send and receive messages was consistently at or above $70 \%$ within the duration of the field trial.

\subsection{Performance Evaluation}

The field trial was conducted over a period of 2 weeks (10 days). Since most villages had power supply for a maximum of $5 \mathrm{hrs}$ daily (and that too not continuously), the rest of the time the Kiosks were powered by a diesel generator. The software installation of the 5 Kiosks \& $\mathrm{BoB}$ and training of the Kiosk operators were all completed within a period of $6 \mathrm{hrs}$.

The performance of the messaging system was observed to be in par with our expectations. The analysis showed the message delivery mechanism to be very reliable with each message being delivered to the correct Kiosk every time the BoB established a $\mathrm{WiFi}^{\mathrm{TM}}$ connection with it. The inherent retry mechanism of SMTP added an extra level of robustness to the delivery system. However, the routing algorithm of [9] was not tested extensively, as we had a single up-down route, with no BoB-to-BoB transfers.

The biggest bottleneck we observed was the BoB, which had limited processing power, buffer size and flash write speed leading to occasional TCP window overflows, specially when a large number of messages were transferred from the Kiosk to the BoB. This result was to be expected mainly because of the 
unbalanced communication equipment used on the 2 sides: a PC as a Kiosk and a mobile phone as a BoB. This problem was partially mitigated after the field trial by reducing the maximum number of simultaneous SMTP sessions to 8 (from 20) when the Kiosk transferred messages to the $\mathrm{BoB}$. The limitation on the disk space available on the $\mathrm{BoB}$ was overcome by ensuring halfduplex message transfers: the BoB was first allowed to transfer all messages intended for the Kiosk it was connected to prior to receiving messages from the Kiosk for delivery along its route. Extending the light weight control protocol to manage the SMTP session scheduling would really help optimize the message transfer between the Kiosk and the BoB.

The time taken to deliver messages from one village Kiosk to another was between $10 \mathrm{mins}$ to $20 \mathrm{mins}$. A peak $\mathrm{WiFi}^{\mathrm{TM}}$ transfer rate of about $6 \mathrm{Mbps}$ was measured, close to the theoretical throughput limit of $6.5 \mathrm{Mbps}$. Assuming a maximum disk space of $10 \mathrm{MB}$, the bandwidth of the data channel between two neighboring villages on the bike route, say, between Village D \& Village E (15 min data travel time plus 2 min of data transfer time at each Kiosk) was estimated to be roughly $70 \mathrm{Kbps}$, which could be further increased by making more disk space available on the $\mathrm{BoB}$ for data storage.

\section{DTN DEPLOYMENT CHALLENGES}

The research challenges in deployment of such a complex system like MotoPost ${ }^{\mathrm{TM}}$ could be broadly described to fit into two categories: Socio-Economic and Technological.

\subsection{Socio-Economic}

During the field trial it was observed that the trust with the operator of any service is an important factor in the uptake/usage of a service offered, which was also reported in the user studies of [7]. The choice of a local entrepreneur to run the Kiosk helped to soften the interactions with the local population. Another major factor was privacy, as people prefer systems and options that ensure that they are in control of sharing their information, and they also want to be sure that no one is snooping on their exchanges. These aspects need to be incorporated while designing any communication system for these communities. Also unlike a government run postal service, the MotoPost ${ }^{\mathrm{TM}}$ Kiosk could be made operational during non-wage earning hours that are convenient to most rural folks (typically early hours or late hours). There is a possibility of hosting other services (retail of physical goods, etc.,) in a Kiosk, which can be explored further.

The social impact of using synthetic media or avatar to guide the user versus using a Kiosk operator needs to be explored further. The field trial indicated that an avatar based user interface does help in improving usability of the system.

The other issue that needs to be explored is the use of a mobile phone as a Kiosk as opposed to a fixed Kiosk location.

Any network deployment should also take into account multiple stake holders and reach service level agreements for sustainable operations of a system like MotoPost ${ }^{\mathrm{TM}}$. The operator running such a network may choose to sign different types of service agreements with bus operators, public or private, depending on, for example, the routes they take, the distance covered by each route, the frequency of the buses, the reliability guarantee offered by each bus operator, etc. for information transfer in the system. Creating a set of services that would sustain Kiosk operations is another area that needs further investigation; there have been recent studies which looked into issues involved in creating a sustainable kiosk operation.

\subsection{Technology}

Some of the technological challenges in deploying such a network are listed below.

Synchronization on a Delay Tolerant network: There are a few elements in the network that have to be synchronized across elements, some of them are critical like time for e.g. and the others would be application specific like user directory, new user updates, new security parameters, network topology changes etc... The issue of propagating data updates in such a network needs to be addressed.

Provisioning in a DTN Network: A typical DTN network would have to be designed to be self configurable, however there are parameters like routing tables, security parameters that would have to be updated during the operation of the network. Manual configuration would not be scalable and is also prone to misconfiguration errors. Some of the future work needs to investigate on defining new or adapting existing provisioning mechanisms for such networks. The ability to centrally distribute applications or enable internet applications is another example of what a provisioning mechanism would need to address.

Application framework support: During the field trial several applications were highlighted as desirable, some of these applications would need further analysis to support them on a delay tolerant network. One such applications was a channel application were people would subscribe to specific information feeds, such an application would need a definition for a publish/subscribe mechanism on such a network and in addition the publish mechanism would have to be supported by a broadcast/multicast protocol definition on a DTN. Since the data mules are constrained devices, duplicate packets and information on these devices need to be avoided at all costs. The last hop routing and delivery algorithms would need to be redefined to support this.

Another class of applications that need true off-lining support include form filling applications, in some cases there is added complexity of handling Ajax support.

Constraint based routing and route forwarding: Routing is a critical issue especially when you have resource constrained data mules. Routing and route forwarding algorithms have to be developed taking into account constraints of cost, available memory and also time to provide an optimal service quality appropriate with the premium paid for the service. Network architecture plays an important role in addressing and route forwarding, and in MotoPost ${ }^{\mathrm{TM}}$ we chose a hierarchical approach with the belief that a hierarchical approach was best suited for such a geographically distributed network. But the maximum number of hierarchical elements is still a matter of further investigation.

Recovery and self healing mechanisms: The data mule was a dual-mode cell phone, which could be transported using any means. Ultra mobile devices are prone to be lost or damaged; efficient recovery mechanisms should be built to ensure that messages that are stored on these devices are not permanently lost and that the network can recover from the loss of any network element.

Here are highlighted some of the challenges which need to be addressed for a large scale deployment of a DTN network. A deployment should ensure that the network operation is profitable to all parties involved and also ensure that the end consumer gets appropriate value at the right price. In addition the technology 
issues need to be addressed to ensure that the network functions smoothly with a desired service quality.

\section{CONCLUSIONS}

DTN is a promising technology that can extend networking into connectivity challenged regions, but there are specific socioeconomic and technology issues which need to be credibly addressed before deploying such systems in a large scale.

In the trial MotoPost ${ }^{\mathrm{TM}}$ system, the focus was on providing a picture and voice modality messaging services, with a message size limitation of $250 \mathrm{~KB}$. At this size, and with the type of alternative connectivity options that were available during the field trial, we conclude that use of SMTP based message delivery is adequate to transfer such messages in a reliable manner across the network.

The PIN based addressing mechanism described in this paper, helped us map geographical locations to logical node addresses and hence simplified message transfer and routing. We plan to conduct further research to extend this hierarchical subnet based routing algorithms and also look into provisioning [9].

In future, as the availability of a much wider variety of transport \& data-link opportunities increase, each with its own diverse reliability \& throughput characteristics, at different regions \& times throughout the network the applicability of this technology needs to further investigated.

\section{ACKNOWLEDGEMENTS}

Our sincere thanks go to Aman Sagar \& Dhaval Joshi for designing the MotoPost ${ }^{\mathrm{TM}}$ User Interface. We also thank Prashant Joshi who helped us with the collection of system data used in this paper.

\section{REFERENCES}

[1]. Brewer, E., Demmer, M., Du, B., Ho, M., Kam, M., Nedevschi, S., Pal, J., Patra, R., Surana, S. and Fall, K. "The Case for Technology in Developing Regions". IEEE Computer, vol. 38, no. 6, May 2005, pp. $25-38$.

[2]. Brewer, E., Demmer, M., Ho, M., Honicky, R.J., Pal, J., Plauche, M. and Surana, S. "The Challenges of Technology Research for Developing Regions". IEEE Pervasive Computing, vol. 5, no. 2, Apr-Jun 2006, pp. 15-23.

[3]. Cerf, V., Burleigh, S., Hooke, A., Togerson, L., Durst, R., Scott, K., Fall, K. and Weiss, H. "Delay Tolerant Networking Architecture”. RFC 4838, Apr. 2007.

[4]. DTN Research Group http://www.dtnrg.org/

[5]. IndiaPost, Indian Department of Posts. http://www.indiapost.gov.in/Pincode.html

[6]. Pentland, A., Fletcher, R. and Hasson, A. "DakNet: Rethinking Connectivity in Developing Nations". IEEE Computer, vol. 37, no. 1 Jan. 2004, pp. 78-83.

[7]. Seshagiri, S., Sagar, A., and Joshi, D. "Connecting the 'Bottom of the Pyramid' - An Exploratory Case Study of India's Rural Communication Environment". Proc. WWW2007, Banff, Canada, May 8-12, 2007.

[8]. Seth, A., Kroeker, D., Zaharia, M., Guo, S. and Keshav, S. "Low-cost Communication for Rural Internet Kiosks using Mechanical Backhauls". Proc. MobiCom 2006, Los Angeles, USA, Sep. 23-26, 2006. DOI= http://doi.acm.org/10.1145/1161089.1161127

[9]. Sen, M., Chintada, S., Raghavan, S., and Naidu, S. "A System to Establish an Affordable Communications Infrastructure in Rural India”. Technical Report. Motorola Technical Publications Library, Jun. 2008. http://techpubs.motorola.com/IPCOM/172154. 